A 50 year old HIV-positive British heterosexual male presented after returning from Thailand. He had developed a tender swollen left wrist. Urine NAAT for CT/GC was negative. He reported condomless oral and vaginal sex with multiple Thai females. Gonococcal tenosynovitis was suspected and extragenital NAATs and cultures for CT/GC were taken; NAAT for pharyngeal gonorrhoea was positive. Single dose ceftriaxone and azithromycin was prescribed, followed by cefixime for 1 week. Two weeks later his symptoms cleared.

Conclusion Reflecting on these cases a DGI diagnosis was attained following careful consideration of possible differentials and persistence in identifying Neisseria gonorrhoeae. Both diagnoses would have been missed if following current testing guidance which recommends penile-only sampling of heterosexual men.

\section{C3 SYPHILITIC AORTITIS IDENTIFIED IN A PATIENT NEWLY DIAGNOSED WITH HIV - THE EMERGING TIP OF AN ONCOMING ICEBERG?}

${ }^{1}$ Noel B Connolly*, ${ }^{1}$ Jonathan Shaw, ${ }^{2}$ Cara Hendry, ${ }^{1}$ Margaret Kingston. ${ }^{1}$ Manchester Centre for Sexual Health, The Hathersage Centre, Manchester Royal Infirmary, 280 Upper Brook Street, Manchester, UK; ${ }^{2}$ Manchester Heart Centre, Manchester Royal Infirmary, Oxford Road, Manchester, UK

\subsection{6/sextrans-2015-052126.36}

Background A 38 year old man presented for HIV testing following his male partner's diagnosis. Examination revealed systolic and decrescendo diastolic heart murmurs, palpable thrill, bounding pulses, and positive Corrigan's sign. He had not tested previously for HIV or syphilis and had been in a monogamous relationship for 8 years. We describe this man who was asymptomatic - from both HIV and aortic valve disease - with incidental diagnosis of severe syphilitic aortitis following partner notification for HIV.

Results HIV antibody test was positive with baseline viral load 239505 copies/ml and CD4 count 103 cell $/ \mu \mathrm{L}$ (8\%). Syphilis serology was positive with rapid plasma reagin (RPR) 1:4. CXR was unremarkable. ECG was consistent with left ventricular hypertrophy with strain. Echo revealed severe mixed aortic valve disease, left ventricular hypertrophy, good LV systolic function and normal aortic arch appearance. He commenced prednisolone $60 \mathrm{mg}$ OD for $5 \mathrm{~d}, 72 \mathrm{hr}$ before starting three weekly doses of 2.4 MU benzathine penicillin. He was admitted for $48 \mathrm{hr}$ for cardiac monitoring at the start of treatment - which proceeded with no complication. Multidisciplinary involvement with GU physicians, cardiologists and cardiothoracic surgeons was instigated from the start with aortic valve \pm root replacement planned imminently.

Discussion Resurgence of syphilis in the UK was reported in the late 1990s with an ongoing epidemic since, mainly involving MSM. Cardiovascular syphilis typically occurs 15-30 years following primary infection with Treponema pallidum, with complications in $10 \%$ of cases. Could this man be amongst the first cases to develop tertiary syphilis in this latest epidemic?

\section{C4 A COMPLICATED CASE OF CANDIDA}

Fiona Cresswell, Catherine Kirby*, Deborah Williams. Brighton and Sussex University Hospital, Brighton, UK

10.1136/sextrans-2015-052126.37
Background Vulvovaginal candidiasis (VVC) is a common condition caused by Candida albicans in 80-92\%. Candida robusta is rarely identified in humans and has only been reported as a cause of VVC in pregnant women. We present a case of chronic Candida robusta VVC.

Case A 25 year-old, on Cerazette, presented to her GP with discharge and vulval itching; treatment with clotimazole was effective but symptoms recurred. In clinic, one month later, a clinical and microscopic diagnosis of VVC was made, she was treated with fluconazole plus econazole pessary and cream. HIV, syphillis, gonorrhoea and chlamydia were negative.

Despite initial improvement she represented with recurrent symptoms, microscopy and culture again confirmed Candida species. Following a fourth presentation oral fluconazole $150 \mathrm{mg}$ every $72 \mathrm{~h} \times 3$ followed by a weekly dose for three months was commenced. She was asymptomatic during this time but relapsed on discontinuation. Microscopy again confirmed spores and on speciation Candida robusta sensitive to fluconazole was isolated. A second 3-month fluconazole course was given. She had now developed provoked vulvodynia. Low-grade symptoms persisted and Candida robusta was again cultured, now resistant to fluconazole. A one-week course of oral voriconazole was given. Follow-up microscopy was negative but her vulvodynia had worsened. Treatment with amitriptyline was commenced and on review two months later culture remained negative and her vulvodynia had improved.

Discussion We report a case of chronic Candida robusta VVC in a non-pregnant immunocompetant woman, which acquired fluconazole resistance and precipitated vulvodynia. Speciation and sensitivity testing are important in women with recurrent symptoms.

\section{C5 A CASE OF REPEATED RHABDOMYOLYSIS ASSOCIATED WITH PEPSE: AN UNCOMMON SIDE EFFECT OF RALTEGRAVIR}

Jennifer Murira*, Sarah Schoeman. The Leeds Centre for Sexual Health, Leeds, UK

\subsection{6/sextrans-2015-052126.38}

Background/introduction The first line regime for PEPSE recently changed to Truvada/Raltegravir. We report on a case of rhabdomyolysis associated with Raltegravir.

Case A 25 year old MSM requested PEPSE in February 2013. Commencing Truvada/Kaletra, he switched to Truvada/Darunavir/Ritonavir due to arthralgia. He further received Truvada/Darunavir/Ritonavir 4 months later for another PEPSE request.

A third PEPSE episode was initiated in September 2014 commencing Truvada/Raltegravir. Baseline investigations showed an eGFR $75 \mathrm{ml} / \mathrm{min} / 1.73 \mathrm{~m}^{2}$. Two weeks later the patient was complaining of severe myalgia/lethargy. Also he noticed his urine colour change to brown. Repeat investigations were: creatinine $121 \mathrm{umol} / \mathrm{L}$, eGFR $62 \mathrm{ml} / \mathrm{min} / 1.73 \mathrm{~m}^{2}$, Creatine Kinase (CK) $1392 \mathrm{iu} / \mathrm{L}$, urine protein/creatinine (uPCR) $2.9 \mathrm{mg} / \mathrm{mmol}$. On urgent review he was admitted for IV rehydration and cessation of PEPSE having developed an acute kidney injury and rhabdomyolysis. His CK fell following fluid replacement.

In November our patient was seen again having self-initiated PEP following a needle-stick injury from a used needle. He had taken 1 Truvada/Raltegravir from left over medication. However he had recurring myalgia and lethargy. His repeat CK was 2625 $\mathrm{iu} / \mathrm{L}$. The regime was immediately stopped, however his muscle pains and weakness continued for 3 weeks with a slow decline in his CK. 
It was thought the 2 episodes of rhabdomyolysis were drug related secondary to his PEP regime with Raltegravir.

Discussion/conclusion Myopathy and rhabdomyolysis have been reported with use of Raltegravir, our case highlights a cautionary note in a regime that will become more common place.

\section{Undergraduate Presentations: $3^{\text {rd }}$ June 2015}

\section{U1 ASYMPTOMATIC LYMPHOGRANULOMA VENEREUM IN KNOWN HIV POSITIVE MSM: IS IT MORE COMMON THAN WE THINK?}

${ }^{1}$ Daniel Ward*, ${ }^{2}$ Meg Boothby, ${ }^{2}$ Penny Goold, ${ }^{2}$ Emma Hathorn. ${ }^{1}$ University of Birmingham, Birmingham, UK; ${ }^{2}$ Whittall Street Clinic, University Hospitals Birmingham NHS Foundation Trust, Birmingham, UK

\subsection{6/sextrans-2015-052126.39}

Background/introduction The primary manifestation of lymphogranuloma venereum (LGV) infection in men who have sex with men (MSM) in the United Kingdom (UK) is haemorrhagic proctitis with very low levels of asymptomatic infection reported.

Aim(s)/objectives To evaluate LGV infection in MSM attending a large inner city sexual health and human immunodeficiency virus (HIV) clinic.

Methods Data was retrospectively collected on all MSM diagnosed with rectal Chlamydia trachomatis (CT) from $1^{\text {st }}$ October 2010 to $30^{\text {th }}$ June 2014. Information was collected on presentation, LGV diagnosis, HIV status, concurrent sexual infection, treatment and sexual contacts.

Results 583 MSM had a new diagnosis of rectal CT during the study period of which 173 (29.7\%) were known to be HIV positive. 118 MSM (20.2\%; 64 HIV negative; 54 HIV positive) underwent additional testing for LGV and 32 infections (26 HIV positive MSM) were confirmed. All asymptomatic LGV infections ( $\mathrm{n}=5 ; 15.6 \%)$ were diagnosed in HIV positive MSM whilst all HIV negative MSM with LGV infection were symptomatic.

Discussion/conclusion We report a higher incidence of asymptomatic LGV infection in MSM than previously reported. Whilst the number of confirmed LGV infections is low, results suggest screening for LGV infection may be appropriate in all HIV positive MSM with confirmed rectal CT regardless of symptomatology.

\section{U2 WHAT DO MEN WHO HAVE SEX WITH MEN (MSM) TAKING POST-EXPOSURE PROPHYLAXIS (PEP) FOR HIV FOLLOWING SEXUAL EXPOSURE REPORT ABOUT THEIR RECENT SEXUAL RISK-TAKING BEHAVIOUR?}

Joanna Moore*, Alex Pollard, Carrie Llewellyn. Brighton and Sussex Medical School, Brighton, UK

\subsection{6/sextrans-2015-052126.40}

Background/introduction High-risk sexual behaviour plays a significant role in the increasing incidence of HIV infection among men who have sex with men (MSM) in the UK, despite the availability of post-exposure prophylaxis following sexual exposure (PEPSE).

Aim(s)/objectives Behavioural interventions to encourage safer sexual practices need to be effective and acceptable for their target population. Therefore, this study aims to identify the attitudes and interpretation of risk of MSM taking PEPSE.

Methods Data was collected as part of an ongoing randomised controlled trial evaluating a psychological intervention in reducing risk behaviour amongst MSM prescribed PEPSE. The intervention group received two 30-minute telephone interventions implementing augmented motivational interviewing. In this study, 30 participants were selected from the intervention arm and their interventions analysed for thematic content.

Results Themes included: circumstances of event that led to PEPSE; participant's interpretation of risk; emotions associated with risk; disclosure of HIV status; value attributed to consequences of risk; and reason for seeking PEPSE.

Discussion/conclusion Risks were mostly reported in the context of unprotected anal intercourse (UAI) with casual partners, without discussion of HIV status. One theme that arose was the use of mobile-phone applications to seek casual sexual partners. Reasons given for engaging in UAI included anxiety over suggesting condom use, engaging in UAI as a form of "self-harm", and alcohol intoxication. Concern about the morbidity and stigma associated with HIV and the desire for relationships were motivating factors for avoiding HIV. PEPSE was frequently described as an insight into life-long antiretroviral therapy for HIV infection.

\section{U3 DEMOGRAPHIC ASSOCIATIONS WITH GONORRHOEA INFECTIONS IN BRIGHTON}

1,2Lauren Amor*, ${ }^{2}$ Fiona Cresswell, ${ }^{2}$ Angela Dunne, ${ }^{2}$ Gillian Dean, ${ }^{2}$ Joanna Peters, ${ }^{2}$ John Paul. 'Brighton and Sussex Medical School, Brighton, UK; ${ }^{2}$ Brighton and Sussex University Hospitals, Brighton, UK

\subsection{6/sextrans-2015-052126.41}

Background/introduction Gonorrhoea is a public health problem due to rising incidence and antimicrobial resistance. Understanding drivers of infection locally is important for planning public health interventions.

Aim(s)/objectives Describe demographics, lifestyle factors and antimicrobial resistance of gonorrhoea infections in Brighton.

Methods A prospective study recruited 121 individuals with gonorrhoea. Participants completed a questionnaire and cultures underwent whole genome sequencing. Data from questionnaires and electronic records were anonymised and analysed.

Results Average age was 33.6 years, 7.4\% were female, 91.3\% were white, $80 \%$ were MSM, $6.3 \%$ bisexual males. $35.9 \%$ of MSM were HIV-positive. In MSM, multisite infection was common. MSM had on average 8 partners in 3 months before diagnosis, compared to 4 for heterosexuals. $71.6 \%$ reported visiting a sauna, sex party or the internet to find partners. Sex under the influence of drugs occurred in $39.1 \%$ of HIV-positive MSM, $36.4 \%$ of HIV-negative MSM and $27.3 \%$ of heterosexuals. Most commonly used drugs were mephedrone by MSM and cocaine by heterosexuals. Condom use was lowest in HIV-positive MSM. Previous STIs were more frequent in HIV-positive MSM, particularly syphilis (55\% vs 9.1\%). 66.9\% were culture-positive. Resistance to $>1$ antibiotic occurred in $34.8 \%$ of HIV-positive MSM, 9.1\% of HIV-negative MSM and 9.1\% of heterosexuals. Discussion/conclusion Condom avoidance, frequent partner change and sex under the influence of drugs are common in both HIV-positive and HIV-negative MSM, raising concerns about HIV transmission. Antibiotic resistance is more common in HIV-positive MSM, concurring with the national surveillance programme. Effective interventions targeting this group are needed. 